\title{
Bioinformatic analyses reveal a distinct Notch activation induced by STAT3 phosphorylation in the mesenchymal subtype of glioblastoma
}

\author{
*Wen Cheng, MD, PhD, $, 1,7$ Chuanbao Zhang, MD, PhD, ${ }^{2,3,7}$ Xiufang Ren, MM, ${ }^{4}$ Yang Jiang, MD, PhD, \\ Sheng Han, MD, PhD, 1,7 Yang Liu, MM, ,,7 Jinquan Cai, MD, PhD, 5,7 Mingyang Li, MM,,3,7 \\ Kuanyu Wang, MM, ${ }^{6,7}$ Yanwei Liu, MD, PhD, ${ }^{2,3,7}$ Huimin Hu, MD, PhD, 2,7 Qingbin Li, MD, PhD, 5,7 \\ Pei Yang, MD, PhD, 2,3,7 Zhaoshi Bao, MD, PhD, ${ }^{3,7}$ and Anhua Wu, MD, PhD ${ }^{1,7}$ \\ ${ }^{1}$ Department of Neurosurgery, The First Hospital of China Medical University; ${ }^{4}$ Department of Pathology, Shengjing Hospital \\ of China Medical University, Shenyang; 'Beijing Neurosurgical Institute, Capital Medical University, Beijing; ${ }^{3}$ Department of \\ Neurosurgery, Beijing Tiantan Hospital, Capital Medical University; ${ }^{7}$ Chinese Glioma Cooperative Group (CGCG), Beijing; \\ ${ }^{5}$ Department of Neurosurgery, The Second Affiliated Hospital of Harbin Medical University, Harbin; and ${ }^{6}$ Department of \\ Neurosurgery, The First Affiliated Hospital of Dalian Medical University, Dalian, China
}

OBJECTIVE Glioblastoma (GBM) is the most common and lethal type of malignant glioma. The Cancer Genome Atlas divides the gene expression-based classification of GBM into classical, mesenchymal, neural, and proneural subtypes, which is important for understanding GBM etiology and for designing effective personalized therapy. Signal transducer and activator of transcription 3 (STAT3), a critical transcriptional activator in tumorigenesis, is persistently phosphorylated and associated with an unfavorable prognosis in GBM. Although a set of specific targets has been identified, there have been no systematic analyses of STAT3 signaling based on GBM subtype.

METHODS This study compared STAT3-associated messenger RNA, protein, and microRNA expression profiles across different subtypes of GBM.

RESULTS The analyses revealed a prominent role for STAT3 in the mesenchymal but not in other GBM subtypes, which can be reliably used to classify patients with mesenchymal GBM into 2 groups according to phosphorylated STAT3 expression level. Differentially expressed genes suggest an association between Notch and STAT3 signaling in the mesenchymal subtype. Their association was validated in the U87 cell, a malignant glioma cell line annotated as mesenchymal subtype. Specific associated proteins and microRNAs further profile the STAT3 signaling among GBM subtypes.

CONCLUSIONS These findings suggest a prominent role for STAT3 signaling in mesenchymal GBM and highlight the importance of identifying signaling pathways that contribute to specific cancer subtypes.

http://thejns.org/doi/abs/10.3171/2015.11.JNS15432

KEY WORDS glioblastoma; The Cancer Genome Atlas; TCGA; subtypes; mesenchymal subtype; signal transducer and activator of transcription 3; STAT3; activation; Notch signaling; oncology

$\mathrm{G}$ LiOBlastoma (GBM) is the most common and deadly form of malignant glioma, with a median survival of only 14 months. ${ }^{38}$ Because of its highly aggressive nature, traditional treatment methods such as resection and radio- and chemotherapy are relatively ineffective. Recent studies focused on gene-based GBM classi- fication have laid the groundwork for better understanding of etiology and improved personalized therapy for patient subgroups.

Based on differences in gene expression, The Cancer Genome Atlas (TCGA) classifies GBMs into classical, mesenchymal, neural, and proneural subtypes, ${ }^{48}$ which

ABBREVIATIONS AKT = protein kinase B; EGFR= epidermal growth factor receptor; GBM = glioblastoma; GO = gene ontology; GSEA = Gene Set Enrichment Analysis; JAK =

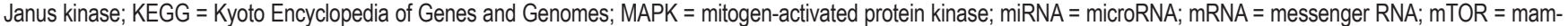
malian target of rapamycin; p-STAT3 = phosphorylated-STAT3; RIPA = radioimmunoprecipitation assay; RNAi = RNA interference; RPPA = reverse-phase protein array; RTK = receptor tyrosine kinase; siRNA = short interfering RNA; STAT3 = signal transducer and activator of transcription 3; TCGA = The Cancer Genome Atlas; TGF- $\beta=$ transforming growth factor- $\beta$.

SUBMITTED February 24, 2015. ACCEPTED November 13, 2015.

INCLUDE WHEN CITING Published online March 11, 2016; DOI: 10.3171/2015.11.JNS15432.

* Drs. Cheng and Zhang contributed equally to this work. 
differ significantly in terms of microenvironment and signaling pathways involved. The mesenchymal subtype is distinguished from the others as being particularly malignant ${ }^{1,10,13,25}$ the overexpression of mesenchymal marker genes is sufficient to induce aggressive behavior in tumors and confer poor prognosis in patients. ${ }^{40}$ We propose that activating a small regulatory module can induce and maintain a specific phenotypic state in glioma cells. For instance, the activation of key proteins may be associated with mesenchymal transformation, which is accompanied by extensive necrosis, angiogenesis, and an enhanced inflammatory/immune response. ${ }^{1,10,13,25}$ However, the factors that specifically induce the mesenchymal subtype are unknown.

Several oncogenic signaling pathways such as protein kinase B (AKT), mitogen-activated protein kinase (MAPK), receptor tyrosine kinase (RTK), and transforming growth factor- $\beta$ (TGF- $\beta$ ) contribute to GBM progression; ${ }^{5,48}$ these converge at pivotal molecular hubs, such as signal transducer and activator of transcription 3 (STAT3). A previous study found that STAT3 induces an aberrant mesenchymal transition in gliomagenesis. ${ }^{6}$ STAT3 can be phosphorylated by several kinases including Janus kinase (JAK) family members, RTKs such as epidermal growth factor receptor (EGFR), and non-receptor-associated tyrosine kinases such as members of the Src family. ${ }^{5}$ In GBM, STAT3 tends to be highly expressed and further constitutively activated compared with normal brain tissue, ${ }^{30}$ thereby maintaining tumor-initiating capacity and modulating the tumor microenvironment. ${ }^{11,41,60}$ In a wide variety of cancers including GBM, aberrant STAT3 signaling confers a negative clinical outcome in patients due to the activation of molecular targets associated with invasion, proliferation, angiogenesis, and immune response. .,29,30,57

The Cancer Genome Atlas contains extensive GBM patient data from reverse-phase protein arrays (RPPAs), messenger RNA (mRNA) microarrays, and microRNA (miRNA) sequence analyses. Data from RPPAs provide information on protein expression and phosphorylation, making it possible to use computational methods to analyze the relationship between a wide range of expression patterns and the activation of specific hub proteins. The present study compared the mRNA, protein, and miRNA expression profiles associated with different STAT3 phosphorylation states across different GBM subtypes.

\section{Methods \\ Patient Samples}

Reverse-phase protein array and mRNA microarray expression and miRNA sequencing data for patients with GBM were downloaded from TCGA database (http:// cancergenome.nih.gov). A total of 178 primary GBM cases with detailed clinical information and corresponding expression data were included in this study. Patients were ranked according to phosphorylated (p)-STAT3 level, with the top and bottom one-third of the list ultimately selected for further analysis and comprising high and low p-STAT3 groups, respectively (60 samples each). The clinical and molecular characteristics of the samples in the 2 groups were compared and are shown in Table 1 . The ethics com-
TABLE 1. Clinical and molecular pathology features of GBM samples associated with the phosphorylation level of STAT3*

\begin{tabular}{|c|c|c|c|}
\hline \multirow[b]{2}{*}{ Variable } & \multicolumn{2}{|c|}{ Level of Phosphorylation } & \multirow[b]{2}{*}{$\mathrm{p}$ Value } \\
\hline & Low & High & \\
\hline Age, yrs & & & $0.913 \dagger$ \\
\hline Mean, range & $60.9,23.1-85.6$ & $60.7,21.7-81.9$ & \\
\hline Sex & & & $1.000 \ddagger$ \\
\hline M & $36(60)$ & $35(58)$ & \\
\hline $\mathrm{F}$ & $24(40)$ & $25(42)$ & \\
\hline GBM subtype & & & $0.077 \ddagger$ \\
\hline Classical & $10(17)$ & $22(37)$ & \\
\hline Mesenchymal & $16(27)$ & $14(23)$ & \\
\hline Neural & $13(22)$ & $7(12)$ & \\
\hline Proneural & $21(35)$ & $17(28)$ & \\
\hline KPS score & & & $1.000 \ddagger$ \\
\hline$\geq 80$ & $32(68)$ & $31(66)$ & \\
\hline$<80$ & $15(32)$ & $16(34)$ & \\
\hline MGMT promoter status & & & $1.000 \ddagger$ \\
\hline Methylation & $21(46)$ & $23(47)$ & \\
\hline No methylation & $25(54)$ & $26(53)$ & \\
\hline IDH1 mutation & & & $1.000 \ddagger$ \\
\hline Mutation & $4(7)$ & $4(7)$ & \\
\hline Wild-type & $52(93)$ & $50(93)$ & \\
\hline Median OS, days & 255 & 272 & $0.545 \S$ \\
\hline No. of deaths & $47(78)$ & $40(67)$ & $0.220 \ddagger$ \\
\hline
\end{tabular}

$I D H 1=$ isocitrate dehydrogenase 1; KPS = Karnofsky Performance Scale; $M G M T=O(6)$-methylguanine DNA methyltransferase; OS = overall survival.

* There were 60 samples (60 patients) in each phosphorylation group. Values are expressed as the mean (\%), unless otherwise stated.

$\dagger$ Student's t-test.

$\ddagger \chi^{2}$ test or Fisher's exact test.

$\S$ Log-rank test.

mittee of The First Hospital of China Medical University approved this study.

\section{Expression Data Analysis}

Expression data were analyzed based on a previously published method applied to breast cancer data. ${ }^{47}$ First, mRNA microarray data for high and low p-STAT3 expression were compared with the Student t-test in each GBM subtype to identify genes that are differentially regulated by STAT3 signaling. The top 300 genes were imported for consensus clustering and were hierarchically clustered with the complete linkage method, using Cluster 3.0. The association between the expression of p-STAT3 and other proteins was evaluated by Pearson correlation analysis for each GBM subtype. All available protein data were depicted as a heat map based on relevant correlation coefficients, and only data with the highest correlation were plotted and used for comparisons between sample subgroups. Finally, the Student t-test was also used to identify miRNAs that were differentially expressed between patients with high and low p-STAT3 expression in each subtype. Based on differentially expressed miRNAs, 
an miRNA coexpression network in mesenchymal GBM was established with a threshold correlation coefficient of 0.6, using Qlucore.

\section{Gene Ontology and Kyoto Encyclopedia of Genes and Genomes Pathway Analysis Using DAVID and Gene Set Enrichment Analysis}

After comparing mRNA expression, differentially expressed genes were subjected to gene ontology (GO) analysis using the Database for Annotation, Visualization and Integrated Discovery (DAVID) (http://david.abcc.ncifcrf. gov/home.jsp) functional annotation tool. ${ }^{18}$ To obtain more information on the relationship between Notch and STAT3 signaling, Gene Set Enrichment Analysis (GSEA) (http:// www.broadinstitute.org/gsea/index.jsp) was performed to determine whether the identified set of genes showed statistically significant and concordant differences between high and low p-STAT3 states in the mesenchymal GBM subtype based on the C2 Kyoto Encyclopedia of Genes and Genomes (KEGG) gene set collection. ${ }^{45}$

\section{Cell Lines and Cell Culture}

The human glioma cell line U87 was obtained from the Institute of Biochemistry and Cell Biology. The U87 cell line was cultured in Dulbecco's modified Eagle's medium supplemented with 10\% fetal bovine serum (Invitrogen) and antibiotics (penicillin and streptomycin). Cultures were incubated at $37^{\circ} \mathrm{C}$ in a humidified chamber with $5 \%$ $\mathrm{CO}_{2}$. The microarray-based expression profile of the U87 cell line was downloaded from Cancer Cell Line Encyclopedia (http://www.broadinstitute.org/ccle/home). The data were classified according to the Proneural-neural-classical-mesenchymal classes using the signatures published ${ }^{48}$ and single-sample GSEA algorithm. The result showed that U87 had a predominant feature of mesenchymal tumors.

\section{Oligonucleotides and Cell Transfection}

Specific short interfering RNA (siRNA) sequences targeting STAT3-376i (5'-GGCCAGCAAAGAAUCACAU

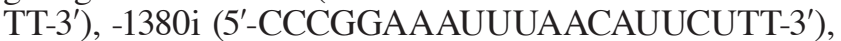
-1837i (5'-GGGACCUGGUGUGAAUUAUTT-3'), and a negative control (5'-UUCUCCGAACGUGUCACGUTT$3^{\prime}$ ) were obtained from GenePharma. For the siRNA experiments, U87 cells were transfected with either a control (nontargeting human RNA) or an siRNA against STAT3 at a final concentration of $200 \mathrm{nM}$. All cell transfections were introduced by the RNA interference (RNAi) MAX reagent (Invitrogen) according to the manufacturer's instructions. The cell transfections were performed in 6-well plates. For each transfection, $7 \mu \mathrm{l}$ of transfection reagent was used and 3 replication experiments were performed.

\section{Western Blot}

Whole-cell lysates were prepared using radioimmunoprecipitation assay (RIPA) buffer. Equal amounts of total protein $(30 \mu \mathrm{g})$ from cell lysates were loaded on a $6 \%$ sodium dodecyl sulfate-polyacrylamide gel electrophoresis gel, transferred to a polyvinylidene difluoride membrane (Millipore), and detected using an enhanced chemilumi- nescence Western blotting detection system (Bio-Rad). Primary antibodies were those against STAT3 (ab68153; Abcam; 1:1000), STAT3 (phosphor Y705) (ab76315; Abcam; 1:200,000), NOTCH1 (ab52627; Abcam; 1:1000), LFNG (ab151699; Abcam; 1:1000), and GAPDH (wl01743; Wanleibio; 1:1000). Secondary antibodies used were goat anti-rabbit IgG-HRP (Wanleibio). Immunoblots were quantified using Image Lab 5.1 software (Bio-Rad).

\section{Statistical Analysis}

SPSS software (IBM Corp.) and GraphPad Prism 6 (GraphPad Software, Inc.) were used for statistical analyses. Differences in clinical and molecular pathological features between high and low p-STAT3 groups were evaluated with the Student t-test or the chi-square test. The Student t-test was also used to identify differentially expressed genes and miRNAs regulated by STAT3 signaling. A Pearson correlation analysis was used to assess the association between the expression of p-STAT3 and other proteins in each GBM subtype. Statistical significance was defined as a 2-tailed $\mathrm{p}$ value $<0.05$.

\section{Results}

\section{Patient Selection Based on STAT3 Phosphorylation Level}

A total of 178 patients with primary GBM (52 classical, 43 mesenchymal, 29 neural, and 54 proneural cases; Fig. 1A) were included in the analysis. Patients were ranked based on the level of STAT3 tyrosine 705 phosphorylation, ${ }^{5}$ and only those with STAT3 tyrosine 705 phosphorylation ranked in the top and bottom one-third of the list (high and low p-STAT3, respectively) were ultimately selected (Fig. 1B) and categorized based on GBM subtype. Among 52 patients with classical GBM, 10 had high and 22 had low p-STAT3; among 43 patients with mesenchymal GMB, 16 had high p-STAT3 and 14 had low p-STAT3. Among 29 patients with neural GBM, 13 had high and 21 had low p-STAT3; and among 54 patients with proneural GBM, 7 had high and 17 had low p-STAT3. There were no differences between patients with high or low p-STAT3 expression in terms of clinical and molecular pathology features (Table 1).

\section{STAT3 Phosphorylation Stratifies Patients With the Mesenchymal but Not Other GBM Subtypes Into 2 Groups}

Genes that were differentially expressed in the selected patients were identified by comparing microarray data for high and low p-STAT3 cases in each GBM subtype with the Student t-test. Differential expression was determined using a threshold $p$ value of $0.05 ; 1639$ genes in classical, 913 genes in mesenchymal, 916 genes in neural, and 1229 genes in proneural cases met this criterion. Despite the large numbers of genes in each subtype, there was little overlap between them, and no genes were detected in all 4 subtypes, indicating that STAT3 signaling had distinct roles in each of these.

To visualize patterns in the activation of STAT3 signaling, unsupervised hierarchical clustering was performed based on the top 300 differentially expressed genes, ranked according to $\mathrm{p}$ value. In the mesenchymal subtype, apparent clustering pattern stratified patients into 2 groups 

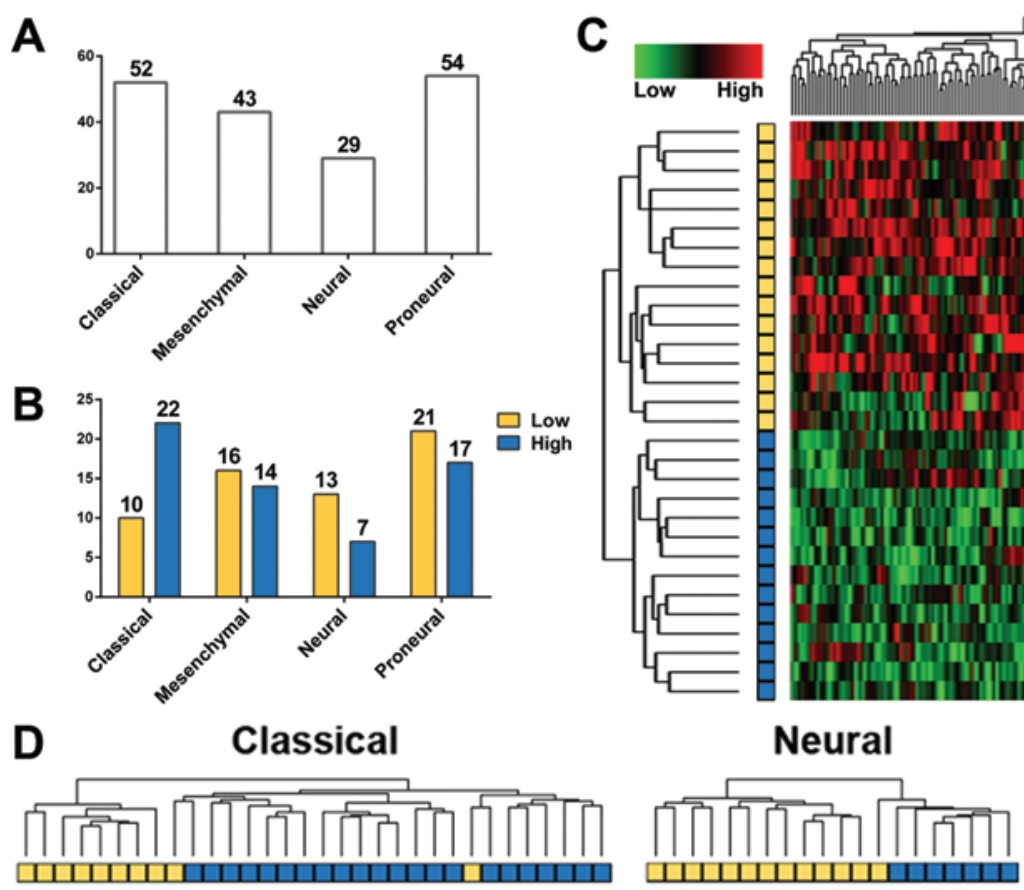

\begin{abstract}
FIG. 1. Clinical and expression data for 178 patients with GBM were available for analysis (A). Patients were ranked according to p-STAT3 expression level, and those in the top and bottom one-third of the list (high and low p-STAT3, respectively) were selected for further analysis (B). A hierarchical cluster analysis based on the top 300 differentially expressed genes revealed a clustering of genes that divided patients into 2 groups according to p-STAT3 level in the mesenchymal (C) but not in the other GBM subtypes (D). High and low p-STAT3 levels are indicated by blue and yellow, respectively. Figure is available in color online only.
\end{abstract}

according to p-STAT3 level (Fig. 1C). In contrast, classifications observed in the other 3 GBM subtypes were unreliable (Fig. 1D). A principal component analysis revealed that differentially expressed genes regulated by p-STAT3 in mesenchymal GBM clearly distinguished patients as having high or low p-STAT3 (Fig. S1), underscoring the critical role of STAT3 signaling in this subtype. (A table or figure designated " $\mathrm{S}$ " is supplemental material available online only; URL is provided in the end matter.) The clinical and molecular pathological features were compared between mesenchymal cases with high and low pSTAT3 (Table S1).

\section{Gene Ontology and KEGG Pathway Analysis of Differentially Expressed Genes Associated With STAT3 Signaling}

Gene ontology and KEGG pathway analyses were performed to compare functional annotations associated with STAT3 signaling across GBM subtypes. We first compared the 913 genes that were differentially expressed between mesenchymal high and low p-STAT3 patients with the 3341 genes that were differentially expressed between high p-STAT3 in mesenchymal and classical subtypes, which yielded a total of 215 common genes (Fig. 2A). Second, the 913 genes that were differentially expressed in the mesenchymal subtype were compared with the 2449 genes that were differentially expressed between mesenchymal and neural high p-STAT3, which comprised 136 overlapping genes (Fig. 2B). Third, a total of 275 differentially expressed genes were found to overlap after compar- ing the 913 genes that were differentially expressed and 3956 genes that were differentially regulated in mesenchymal versus proneural high p-STAT3 (Fig. 2C). Finally, we compared the above-mentioned 215,136 , and 275 genes that overlapped between groups to identify differentially expressed genes that were specific to the mesenchymal high p-STAT3 subgroup, yielding a total of 22 genes (Fig. 2D; Table S2).

To elucidate gene functions and signaling pathways associated with STAT3 signaling, GO and KEGG pathway analyses were performed as follows. The 913 genes that were differentially expressed in the mesenchymal subtype were analyzed first. The most significant biological processes associated with altered STAT3 signaling included cell-cell signaling, ion transport, cell adhesion, and neurogenesis, when ranked according to $\mathrm{p}$ value. Subsequently, a similar analysis was done for the 22 genes identified as being specific to the mesenchymal high p-STAT3 subgroup. Only 2 biological processes reached statistical significance: activation of the Notch signaling pathway and regulation of Notch signaling, with enrichment scores of 41.077 and 129.455 , respectively (Fig. 2E). The mesenchymal subtype of GBM was distinguished by the complexity of biological processes responsible for its highly aggressive nature; through GO analysis, we found that the Notch signaling pathway is associated with STAT3 signaling activation in the mesenchymal subtype. Gene Set Enrichment Analysis was performed to identify other pathways regulated by STAT3 signaling and to validate the association between Notch and STAT3 signaling in the mesen- 

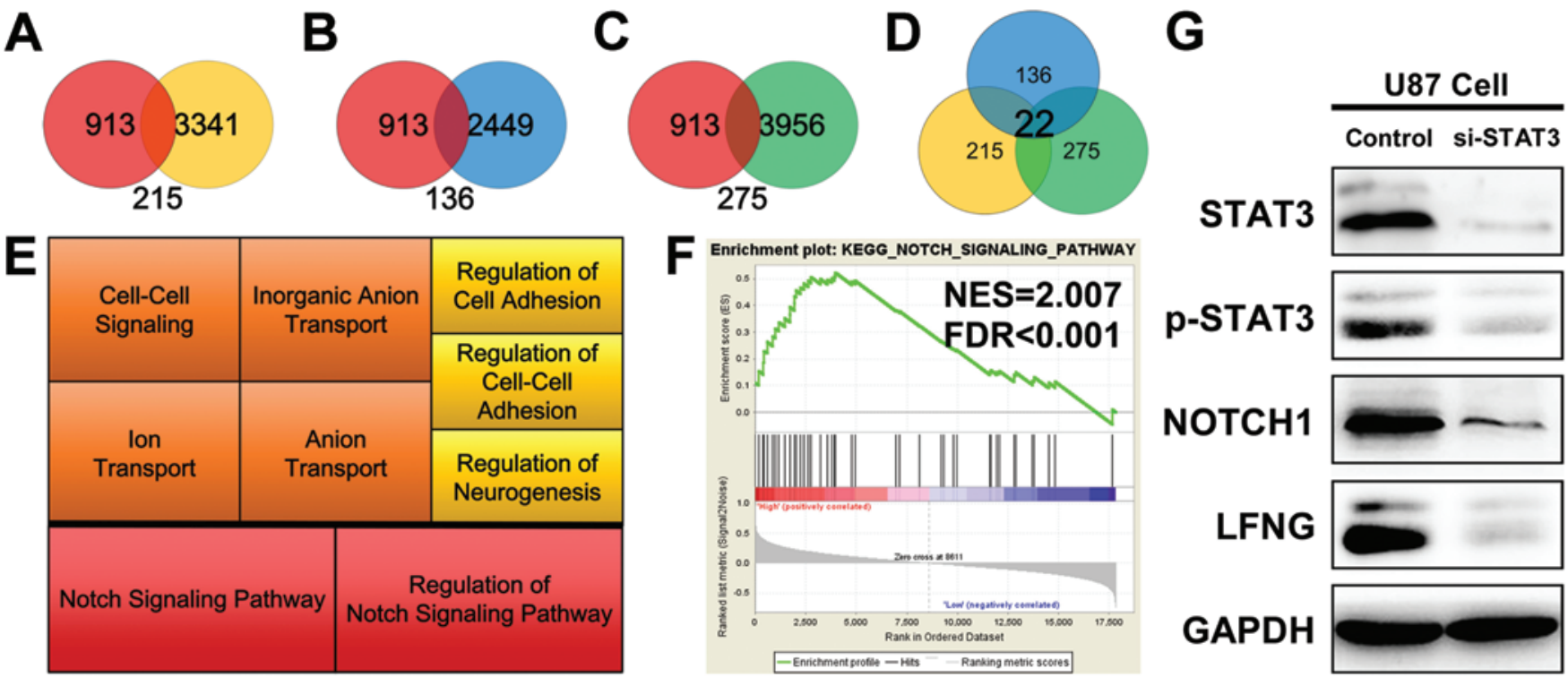

FIG. 2. A total of 913 genes differentially expressed in the mesenchymal subtype were compared with 3341 genes differentially expressed between mesenchymal and classical high p-STAT3 groups, yielding a set of 215 overlapping genes (A); 2449 genes differentially expressed between mesenchymal and neural high p-STAT3 groups, yielding 136 overlapping genes (B); and 3956 genes differentially expressed between mesenchymal and proneural high p-STAT3 groups, yielding 275 overlapping genes (C). A comparison of the 215, 136, and 275 genes revealed 22 common genes specific to the mesenchymal high p-STAT3 subgroup (D). A GO analysis using the DAVID (Database for Annotation, Visualization and Integrated Discovery) functional annotation tool was performed to determine the most relevant biological processes based on the 913 genes that were differentially expressed in the mesenchymal subtype, which are plotted in the upper half of the diagram (E); the most highly correlated processes based on the 22 overlapping genes are plotted in the lower half. The GSEA indicated that Notch signaling was significantly enriched in patients with high p-STAT3 expression in the mesenchymal subtype (F). The U87 cell line was used to validate the signaling association between STAT3 and Notch (G). FDR $=$ false discovery rate; NES = normalized enrichment score. Figure is available in color online only.

chymal subtype. As shown in Table S3, compared with other KEGG annotations, Notch signaling genes were the most highly enriched in mesenchymal high p-STAT3 patients, with a notable normalized enrichment score of 2.007 and false discovery rate $<0.001$ (Fig. 2F).

The microarray-based expression profile of U87 cell line was downloaded from Cancer Cell Line Encyclopedia and genes were classified according to the Proneural-neural-classical-mesenchymal classes, using the signatures published by Verhaak et al. ${ }^{48}$ and single-sample GSEA. The result showed that U87 cell line had a predominant feature of mesenchymal tumors. The cell line, as a mesenchymal tumor model, was selected to test the association between STAT3 and Notch signaling in vitro. The intrinsic STAT3 was knocked down by transfection with a specific siRNA. The loss of expression and phosphorylation of STAT3 was confirmed by Western blot (Fig. 2G). The knockdown of STAT3 led to a significant decrease of NOTCH1 and LFNG expression, which were critical members of the Notch signaling pathway. These results confirmed the tight association between STAT3 and Notch signaling in the mesenchymal subtype.

\section{Differences in Functional Modules Associated With STAT3 Signaling Among GBM Subtypes}

Protein and phosphorylation data from TCGA were analyzed to identify signaling pathways correlated with STAT3 signaling in the GBM subtypes. A Pearson correlation analysis was performed between p-STAT3 and other important proteins in each subtype, and heat maps were generated based on correlation coefficients (Fig. 3AD). In each subtype, STAT3 phosphorylation was most closely correlated with signaling pathways linked to human malignancies. In the mesenchymal subtype, the activation of STAT3 signaling was correlated with $\beta$-catenin, Smad1, and mammalian target of rapamycin (mTOR) (Fig. 3F). The correlation coefficients $>0.6$ indicated a closely positive correlation between these signaling pathways and STAT3 signaling. Associations between these 3 proteins and p-STAT3 in the other 3 GBM subtypes were not significant, demonstrating that STAT3 signaling has a distinct function in the mesenchymal compared with the other subtypes.

In contrast, patients in the other 3 GBM subtype groups differed from the mesenchymal subtype group in terms of STAT3 signaling function. In patients with classical GBM, EGFR Y1068 phosphorylation was the most relevant pSTAT3-associated protein, with a lower correlation coefficient of 0.525 (Fig. 3E). Only 1 protein each showed high correlation (coefficient $>0.6$ ) with STAT3 in the neural and proneural subtypes, which were Y1235 phosphorylated c-Met (Fig. 3G) and T202 phosphorylated MAPK (Fig. $3 \mathrm{H})$, respectively. These results suggest that STAT3 activates different signaling pathways in each GBM subtype.

\section{An miRNA Network Is Associated With STAT3 Signaling in Mesenchymal GBM}

In GBM, miRNAs have characteristic expression pat- 

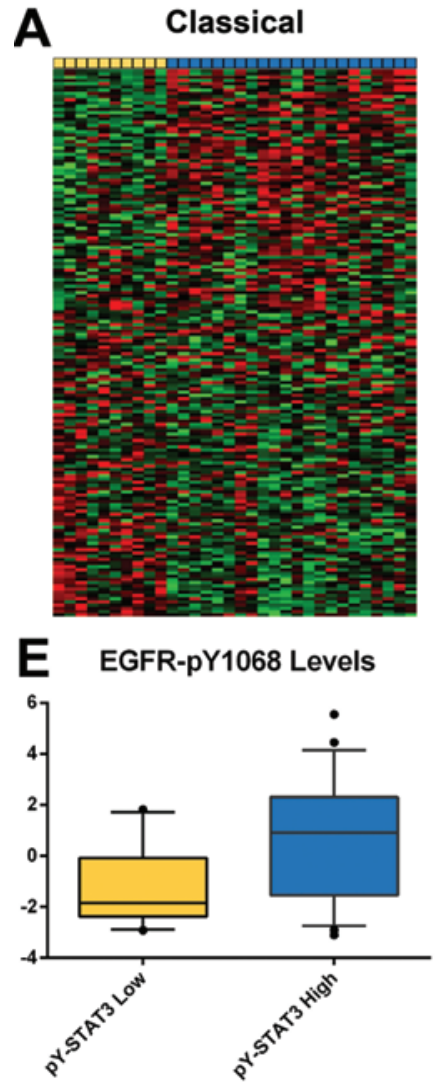
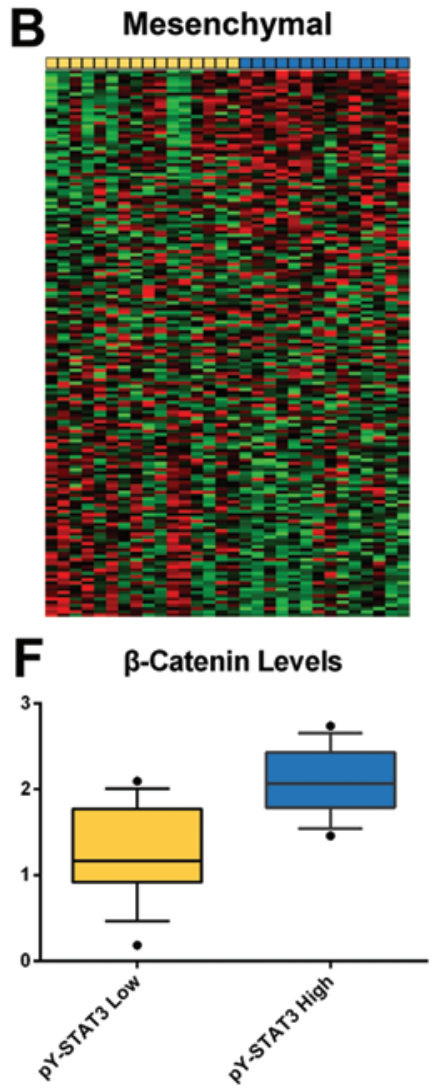
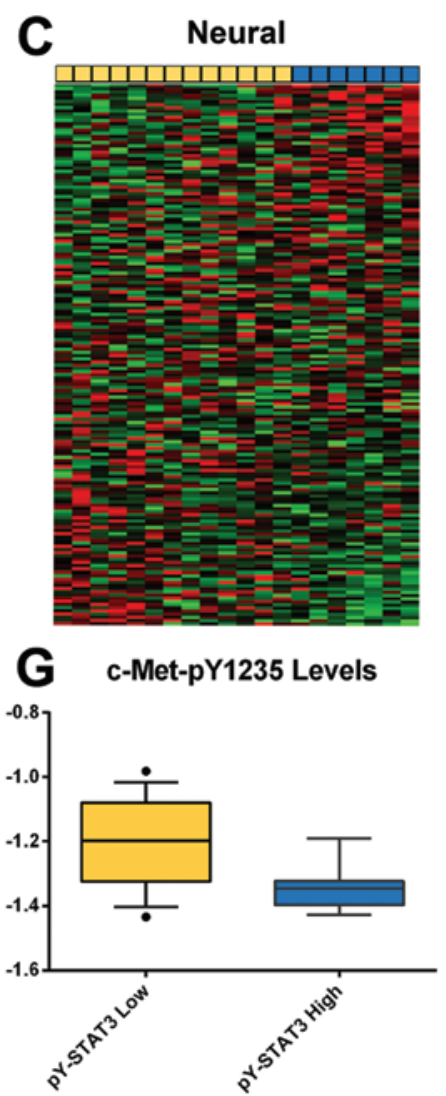

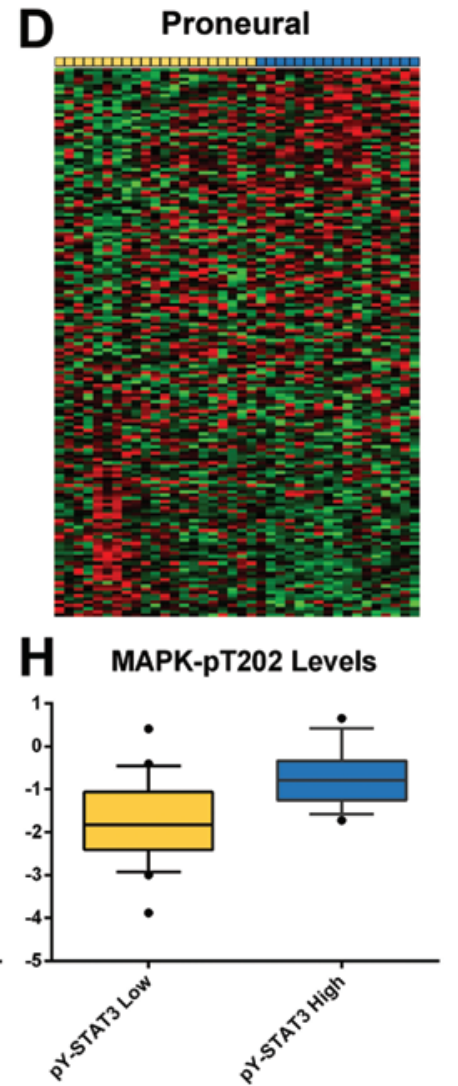

FIG. 3. Protein and phosphorylation data were used to evaluate the association between STAT3 and different signaling pathways according to GBM subtype. To visualize global changes in protein levels associated with STAT3 signaling, heat maps were generated based on correlation coefficients for each subtype (A-D). Protein expression levels with the high correlations were compared between patients with different p-STAT3 levels in each subtype (E-H). High and low p-STAT3 levels are indicated by blue and yellow, respectively. Bars indicate standard error. Figure is available in color online only.

terns and can modulate glioma cell behavior. We sought to identify miRNA networks associated with STAT3 signaling in the GBM subtypes with a focus on the mesenchymal subtype, which is highly associated with STAT3 activity. ${ }^{6}$ We first profiled global miRNA expression by unsupervised hierarchical clustering based on the level of p-STAT3 in each subtype. There were no obvious differences in miRNA expression between high and low STAT3 groups in any of the subtypes, indicating that STAT3 signaling does not strongly affect global miRNA regulation in GBM (Fig. 4A-D).

The miRNA expression profiles of high and low pSTAT3 patients in each subtype were compared with the Student t-test to identify differentially expressed miRNAs associated with STAT3 signaling. In the classical, neural, proneural, and mesenchymal subtypes, $60,35,24$, and 29 miRNAs, respectively, were differentially expressed between high and low p-STAT3 groups (Supplementary Data; see end matter). There was little overlap between them, and no genes were detected in all 4 subtypes. A coexpression analysis of the 29 miRNAs associated with the mesenchymal subtype revealed a coexpression network of 17 miRNAs with a threshold correlation coefficient $>$ 0.6 , indicating a high degree of coexpression (Fig. 4E and Table S4).

\section{Discussion}

Glioblastoma is the most lethal type of glioma. It is often resistant to traditional therapy and invades healthy brain tissue, leading to recurrence after resection. Thus, more effective diagnostic and therapeutic strategies are needed. The classification of GBM into classical, mesenchymal, neural, and proneural subtypes based on gene expression profiles ${ }^{48}$ provides greater understanding of the molecular mechanisms of GBM. We hypothesized that the differential activation of critical signaling pathways induces and maintains each subtype. A previous study reported that STAT3 phosphorylation was the major molecular event associated with mesenchymal transformation in primary GBM. ${ }^{6}$ STAT3 functions in an oncogenic capacity by controlling various cellular processes including proliferation, the immune response, and malignant cell transformation. ${ }^{12}$ However, little is known about the role of activated STAT3 in different GBM subtypes. Numerous upstream and downstream molecules increase the difficulty of using traditional experimental methods to explore the differential role of STAT3 among GBM subtypes. In addition, data on mRNA expression have limited utility for studying signaling pathways that are activated by protein phosphorylation. Information on STAT3 phosphorylation 

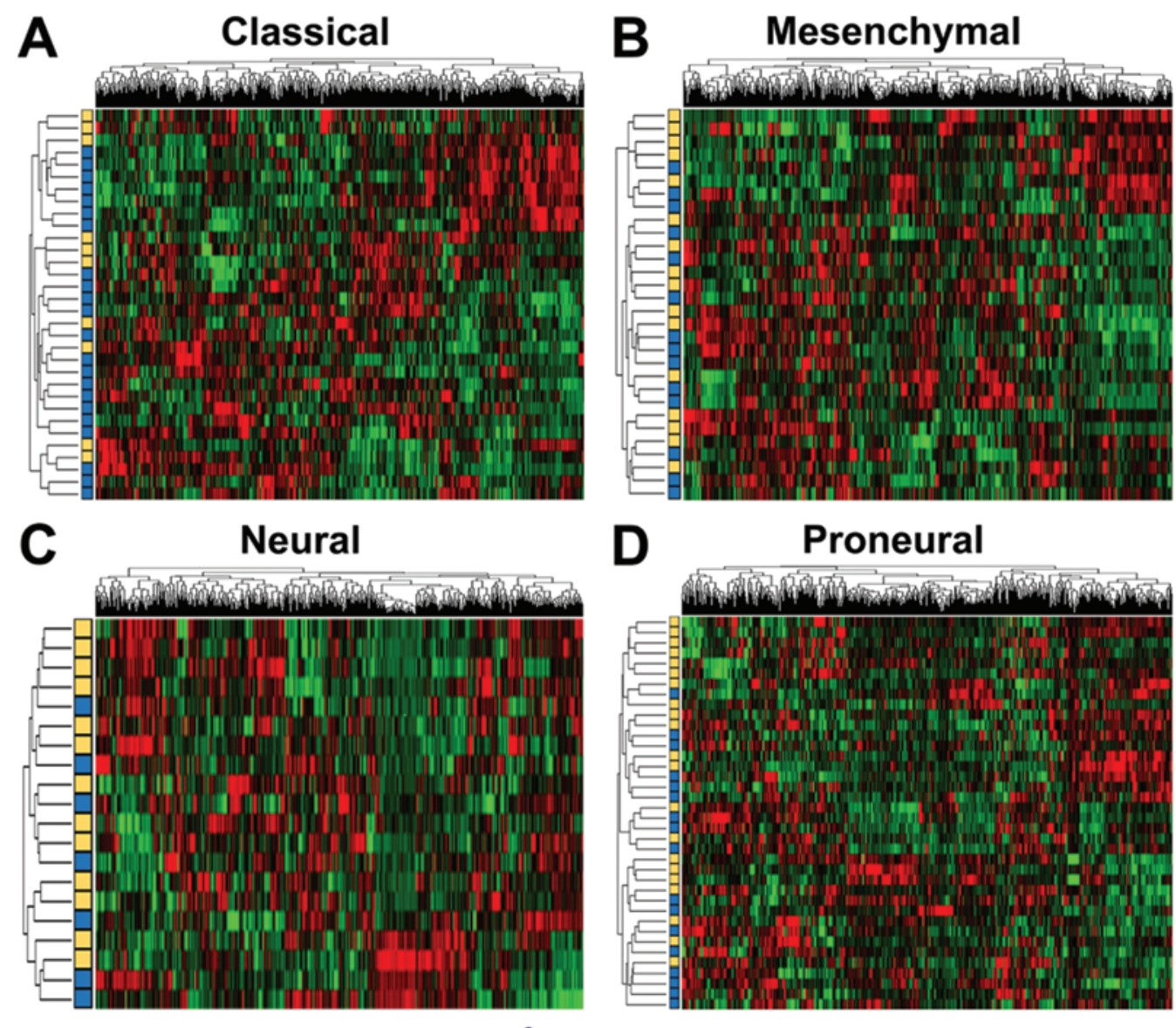

\section{$\mathbf{E}$}
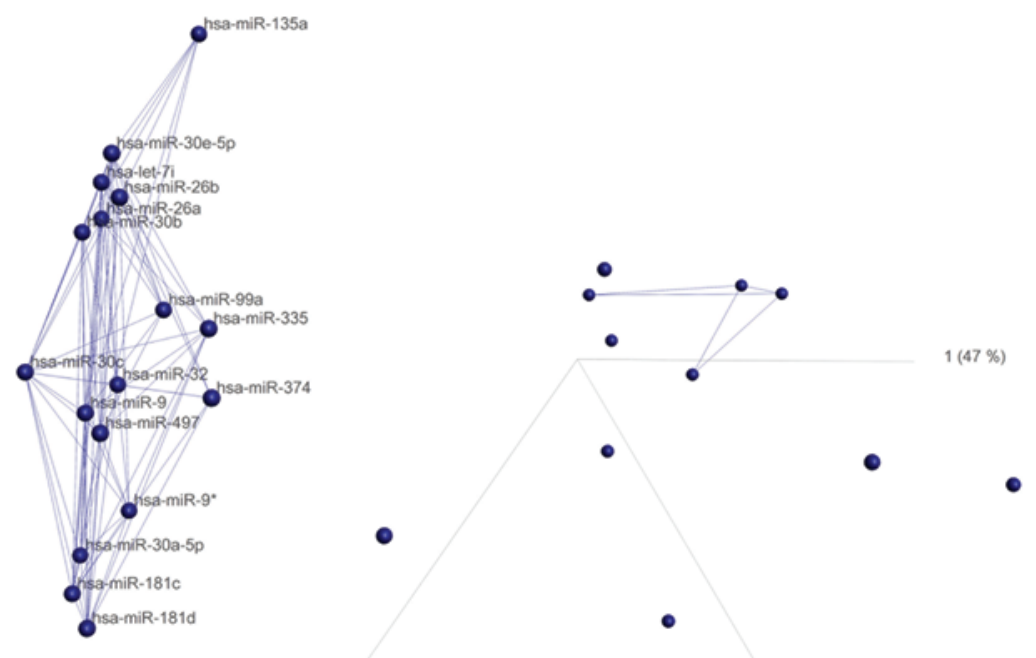

$3(7 \%)$

$\bullet$

FIG. 4. Hierarchical cluster analysis and heat maps showing miRNA sequencing data comparing high and low STAT3 groups in each subtype (A-D). An miRNA coexpression network in patients with mesenchymal GBM was determined based on correlation coefficients $>0.6(E)$. Figure is available in color online only. 
in a large GBM sample is available in TCGA, making it a useful resource for the present study.

We analyzed the role of STAT3 signaling in each GBM subtype and identified subsets of genes that were differentially expressed in each subtype. Interestingly, there was no overlap in the subtype-specific gene sets, indicating that although activated STAT3 signaling induced downstream effects in each subtype of GBM, target genes and mechanisms were unique to each subtype. Strikingly, the classification of patients into 2 groups based on p-STAT3 level was only observed in the mesenchymal subtype. Previous studies have reported that STAT3 controls the expression of other transcriptional regulators and is responsible for the signaling crosstalk that induces the mesenchymal phenotype; ${ }^{6}$ indeed, STAT3 activation has been detected in radiation-induced mesenchymal transition. ${ }^{16}$

In the present study, STAT3 signaling exerted a greater influence on the mesenchymal than on the other subtypes. Moreover, 2 subgroups of patients with mesenchymal GBM were clearly distinguished based on STAT3 activation level, indicating that this pathway plays a key role in defining this subtype. There was no significant difference in clinical and molecular pathological features between these 2 subgroups. However, mesenchymal cases with different levels of STAT3 activation exhibited different expression profiles, suggesting distinct biological conditions.

Although much effort has been made, little progress has been achieved in targeting STAT3 for treatment of GBM. ${ }^{15,19,58}$ It might be attributable to the high heterogeneity of GBM. Considering the prominent role of STAT3 signaling in the mesenchymal subtype, such patients, especially those with highly phosphorylated STAT3, were more likely to benefit from corresponding targeted therapy. Therefore, GBM needs accurate patient classification and selection to fully explore the treatment efficiency. Because STAT3 signaling reduces radio- and chemosensitivity in GBM, ${ }^{39,52}$ targeting STAT3 combined with traditional radio- and chemotherapy could achieve better therapeutic effect.

The mesenchymal phenotype is characterized by the loss of cell adhesion and the activation of specific signaling pathways. ${ }^{14}$ Correspondingly, the GO analysis showed that STAT3 signaling was closely correlated with cell-cell signaling, ion transport, and cell adhesion in the mesenchymal subtype. Moreover, an apparent association between Notch signaling and high level of STAT3 phosphorylation was observed from the GSEA and validated in a mesenchymal glioma cell line, which had not been previously reported. These findings may explain the occurrence of mesenchymal GBM, given the reports that Notch signaling is a key factor specifying cell fate in the developing nervous system ${ }^{2}$ and maintaining the proliferation of neural precursors..$^{17,43}$ Notch signaling also has oncogenic effects, which include stimulation of proliferation, invasion, and gliomagenesis as well as inhibition of apoptosis. Notch signaling is therefore a prognostic marker in glioma. ${ }^{4,31,40}$

Notch signaling may act synergistically with STAT3 in the mesenchymal transition. In a previous study, crosstalk between the 2 pathways was predicted to occur through
Hes proteins, which function as nonreceptor scaffolds that enable the phosphorylation of STAT3 by JAK2..$^{21,36}$ It has been previously reported that along with TGF- $\beta$ and Snail2, Notch triggers the mesenchymal transition in human, murine, and canine cells, which was associated with STAT3 ${ }^{27,59}$ and could be similarly extrapolated to glioma cells. On the other hand, a molecular and phenotypic link has been suggested between mesenchymal transition and chemoresistance in multiple types of cancer cells, including gliomas; ${ }^{42,50,54}$ the adoption of a mesenchymal subtype concomitant with the acquisition of radiation resistance was also observed in GBM. ${ }^{16,35}$ The discovery of reciprocal Notch and STAT3 activity in the mesenchymal transition suggests that targeted pharmacological inhibition of these 2 pathways may overcome chemo- and radiotherapy resistance and lead to better clinical outcomes.

Genetic alterations leading to STAT3 upregulation have yet to be reported, implying that crosstalk with other pathways may be responsible for the abnormal STAT3 activity in different GBM subtypes. ${ }^{33}$ Protein and phosphoprotein data have suggested interactions between STAT3 and $\beta$ catenin, Smad1, and mTOR signaling pathways in mesenchymal but not in other GBM subtypes. This implies that through synergistic combination of subtype-specific mediators, STAT3 levels could be optimal enough to induce transcription of proteins. Targets of $\beta$-catenin include activators of the epithelial-to-mesenchymal transition and STAT3. ${ }^{20}$ The $\beta$-catenin/T-cell factor 4 complex binds directly to the STAT3 gene promoter to activate STAT3 transcription. $^{22,53}$ STAT3 also forms a complex with Smad1 and p300 to induce astrocyte differentiation from neural progenitors via leukemia inhibitory factor and bone morphogenetic protein 2 signaling. ${ }^{37}$

mTOR, an interesting target in GBM, is frequently activated and contributes to the therapeutic resistance phenotype through RTK hyperactivation and inhibition of the tumor suppressor phosphatase and tensin homolog. ${ }^{49}$ The link between the epithelial-to-mesenchymal transition and the activation of mTOR signaling is well established in various cancers. ${ }^{7,8,26}$ mTOR phosphorylates STAT3 at serine 727 , which, in conjunction with phosphorylation at tyrosine 707 , is essential for its maximal activation..$^{56}$ Other studies have proposed that mTOR and Notch interact in reciprocal regulatory loops in an mTOR/STAT3/Notch signaling cascade $;{ }^{34}$ we suggest that it plays a major role in the progression of mesenchymal GBM. Based on this supposition, the cytotoxicity of radiation and temozolomide by targeting mTOR $^{9}$ is probably a result of suppressing the mesenchymal transition.

MicroRNAs have been shown to be associated with STAT3 signaling and GBM progression. ${ }^{46}$ The miRNA networks in which miRNAs can cooperatively regulate gene expression have been given much importance..$^{23,28}$ Therefore, we aimed to profile the STAT3 signaling-relevant miRNA networks. In this study, there were no obvious global changes in miRNA expression in any of the GBM subtypes. However, a small population of miRNAs was associated with STAT3 activation in each subtype, although the functional significance of this observation is unclear. The differentially expressed miRNAs showed no overlap between classical, mesenchymal, neural, and pro- 
neural GBM, implying that a specific set of miRNAs was regulated by STAT3 in each subtype.

A coexpression analysis of 17 differentially expressed and primarily cancer-associated miRNAs was performed in mesenchymal GBM to identify a coexpression network, which was found to include both tumor activators and suppressors. It is urgent to clarify their role as a network in the STAT3 signaling and subtype determination. On the other hand, previous studies have reported tight associations between STAT3 signaling and several miRNAs, such as mir-135a, mir-26a, and mir-9. ${ }^{24,51,55}$ One of the most interesting miRNAs, mir-9, is associated with mesenchymal transition in various cancers including GBM. ${ }^{24,32,44}$ A previous study found that mir-9 suppressed mesenchymal differentiation in GBM by inhibiting the expression of JAK and activation of STAT3. ${ }^{24}$ Consistent with this report, we observed a significant downregulation of mir-9 level in the mesenchymal relative to other GBM subtypes (Fig. S2). Although the interaction between mir-9 and STAT3 in the mesenchymal subtype requires clarification, these findings imply that, as in the case of other regulatory factors, specific miRNAs are essential determinants of GBM subtype.

\section{Conclusions}

The present study revealed the pervasive role of STAT3 signaling in GBM, especially in the mesenchymal subtype. Proteins, mRNAs, and miRNAs associated with STAT3 signaling, as well as cooperation with Notch signaling, were identified specifically in patients with mesenchymal GBM. Based on these findings, we propose that STAT3 has a key role in the development of mesenchymal GBM. Given the poor prognosis and aggressiveness of mesenchymal GBM, it is anticipated that these patients would benefit most from chemotherapeutic strategies that directly or indirectly target STAT3 or related factors.

\section{Acknowledgments}

This work was supported by grants from the National High Technology Research and Development Program of China (863) (2012AA02A508), the National Natural Science Foundation of China (81172409), and the Science and Technology Department of Liaoning Province (2011225034).

\section{References}

1. Arimappamagan A, Somasundaram K, Thennarasu K, Peddagangannagari S, Srinivasan H, Shailaja BC, et al: A fourteen gene GBM prognostic signature identifies association of immune response pathway and mesenchymal subtype with high risk group. PLoS One 8:e62042, 2013

2. Artavanis-Tsakonas S, Rand MD, Lake RJ: Notch signaling: cell fate control and signal integration in development. Science 284:770-776, 1999

3. Birner P, Toumangelova-Uzeir K, Natchev S, Guentchev M: STAT3 tyrosine phosphorylation influences survival in glioblastoma. J Neurooncol 100:339-343, 2010

4. Boulay JL, Miserez AR, Zweifel C, Sivasankaran B, Kana V, Ghaffari A, et al: Loss of NOTCH2 positively predicts survival in subgroups of human glial brain tumors. PLoS One 2:e576, 2007

5. Brantley EC, Benveniste EN: Signal transducer and activator of transcription-3: a molecular hub for signaling pathways in gliomas. Mol Cancer Res 6:675-684, 2008
6. Carro MS, Lim WK, Alvarez MJ, Bollo RJ, Zhao X, Snyder EY, et al: The transcriptional network for mesenchymal transformation of brain tumours. Nature 463:318-325, 2010

7. Chang L, Graham PH, Hao J, Ni J, Bucci J, Cozzi PJ, et al: Acquisition of epithelial-mesenchymal transition and cancer stem cell phenotypes is associated with activation of the $\mathrm{PI} 3 \mathrm{~K} / \mathrm{Akt} / \mathrm{mTOR}$ pathway in prostate cancer radioresistance. Cell Death Dis 4:e875, 2013

8. Chen X, Cheng H, Pan T, Liu Y, Su Y, Ren C, et al: mTOR regulate EMT through RhoA and Racl pathway in prostate cancer. Mol Carcinog 54:1086-1095, 2015

9. Choi EJ, Cho BJ, Lee DJ, Hwang YH, Chun SH, Kim HH, et al: Enhanced cytotoxic effect of radiation and temozolomide in malignant glioma cells: targeting PI3K-AKT-mTOR signaling, HSP90 and histone deacetylases. BMC Cancer 14:17, 2014

10. Cooper LA, Gutman DA, Chisolm C, Appin C, Kong J, Rong $\mathrm{Y}$, et al: The tumor microenvironment strongly impacts master transcriptional regulators and gene expression class of glioblastoma. Am J Pathol 180:2108-2119, 2012

11. Darnell JE: Validating Stat 3 in cancer therapy. Nat Med 11:595-596, 2005

12. de la Iglesia N, Puram SV, Bonni A: STAT3 regulation of glioblastoma pathogenesis. Curr Mol Med 9:580-590, 2009

13. Engler JR, Robinson AE, Smirnov I, Hodgson JG, Berger MS, Gupta N, et al: Increased microglia/macrophage gene expression in a subset of adult and pediatric astrocytomas. PLoS One 7:e43339, 2012

14. Gonzalez DM, Medici D: Signaling mechanisms of the epithelial-mesenchymal transition. Sci Signal 7:re8, 2014

15. Gray GK, McFarland BC, Nozell SE, Benveniste EN: NF-кB and STAT3 in glioblastoma: therapeutic targets coming of age. Expert Rev Neurother 14:1293-1306, 2014

16. Halliday J, Helmy K, Pattwell SS, Pitter KL, LaPlant Q, Ozawa T, et al: In vivo radiation response of proneural glioma characterized by protective p53 transcriptional program and proneural-mesenchymal shift. Proc Natl Acad Sci U S A 111:5248-5253, 2014

17. Hitoshi S, Alexson T, Tropepe V, Donoviel D, Elia AJ, Nye JS, et al: Notch pathway molecules are essential for the maintenance, but not the generation, of mammalian neural stem cells. Genes Dev 16:846-858, 2002

18. Huang W, Sherman BT, Lempicki RA: Systematic and integrative analysis of large gene lists using DAVID bioinformatics resources. Nat Protoc 4:44-57, 2009

19. Jackson C, Ruzevick J, Amin AG, Lim M: Potential role for STAT3 inhibitors in glioblastoma. Neurosurg Clin N Am 23:379-389, 2012

20. Kahlert UD, Maciaczyk D, Doostkam S, Orr BA, Simons $\mathrm{B}$, Bogiel T, et al: Activation of canonical WNT/ $\beta$-catenin signaling enhances in vitro motility of glioblastoma cells by activation of ZEB1 and other activators of epithelial-tomesenchymal transition. Cancer Lett 325:42-53, 2012

21. Kamakura S, Oishi K, Yoshimatsu T, Nakafuku M, Masuyama N, Gotoh Y: Hes binding to STAT3 mediates crosstalk between Notch and JAK-STAT signalling. Nat Cell Biol 6:547-554, 2004

22. Kawada M, Seno H, Uenoyama Y, Sawabu T, Kanda N, Fukui $\mathrm{H}$, et al: Signal transducers and activators of transcription 3 activation is involved in nuclear accumulation of beta-catenin in colorectal cancer. Cancer Res 66:2913-2917, 2006

23. Khan AA, Betel D, Miller ML, Sander C, Leslie CS, Marks DS: Transfection of small RNAs globally perturbs gene regulation by endogenous microRNAs. Nat Biotechnol 27:549555,2009

24. Kim TM, Huang W, Park R, Park PJ, Johnson MD: A developmental taxonomy of glioblastoma defined and maintained by MicroRNAs. Cancer Res 71:3387-3399, 2011

25. Krock BL, Skuli N, Simon MC: Hypoxia-induced angiogenesis: good and evil. Genes Cancer 2:1117-1133, 2011 
26. Lamouille S, Connolly E, Smyth JW, Akhurst RJ, Derynck R: TGF- $\beta$-induced activation of mTOR complex 2 drives epithelial-mesenchymal transition and cell invasion. J Cell Sci 125:1259-1273, 2012

27. Leong KG, Niessen K, Kulic I, Raouf A, Eaves C, Pollet I, et al: Jagged1-mediated Notch activation induces epithelial-tomesenchymal transition through Slug-induced repression of E-cadherin. J Exp Med 204:2935-2948, 2007

28. Li R, Li X, Ning S, Ye J, Han L, Kang C, et al: Identification of a core miRNA-pathway regulatory network in glioma by therapeutically targeting miR-181d, miR-21, miR-23b, $\beta$ Catenin, CBP, and STAT3. PLoS One 9:e101903, 2014

29. Lin GS, Chen YP, Lin ZX, Wang XF, Zheng ZQ, Chen L: STAT3 serine 727 phosphorylation influences clinical outcome in glioblastoma. Int J Clin Exp Pathol 7:3141-3149, 2014

30. Lin GS, Yang LJ, Wang XF, Chen YP, Tang WL, Chen L, et al: STAT3 Tyr705 phosphorylation affects clinical outcome in patients with newly diagnosed supratentorial glioblastoma. Med Oncol 31:924, 2014

31. Lino MM, Merlo A, Boulay JL: Notch signaling in glioblastoma: a developmental drug target? BMC Med 8:72, 2010

32. Lu MH, Huang CC, Pan MR, Chen HH, Hung WC: Prospero homeobox 1 promotes epithelial-mesenchymal transition in colon cancer cells by inhibiting E-cadherin via miR-9. Clin Cancer Res 18:6416-6425, 2012

33. Luwor RB, Stylli SS, Kaye AH: The role of Stat 3 in glioblastoma multiforme. J Clin Neurosci 20:907-911, 2013

34. Ma J, Meng Y, Kwiatkowski DJ, Chen X, Peng H, Sun Q, et al: Mammalian target of rapamycin regulates murine and human cell differentiation through STAT3/p63/Jagged/Notch cascade. J Clin Invest 120:103-114, 2010

35. Meng J, Li P, Zhang Q, Yang Z, Fu S: A radiosensitivity gene signature in predicting glioma prognostic via EMT pathway. Oncotarget 5:4683-4693, 2014

36. Nagao M, Sugimori M, Nakafuku M: Cross talk between notch and growth factor/cytokine signaling pathways in neural stem cells. Mol Cell Biol 27:3982-3994, 2007

37. Nakashima K, Yanagisawa M, Arakawa H, Kimura N, Hisatsune T, Kawabata M, et al: Synergistic signaling in fetal brain by STAT3-Smad1 complex bridged by p300. Science 284:479-482, 1999

38. Ostrom QT, Bauchet L, Davis FG, Deltour I, Fisher JL, Langer CE, et al: The epidemiology of glioma in adults: a "state of the science" review. Neuro Oncol 16:896-913, 2014

39. Ouédraogo ZG, Müller-Barthélémy M, Kemeny JL, Dedieu V, Biau J, Khalil T, et al: STAT3 serine 727 phosphorylation: a relevant target to radiosensitize human glioblastoma. Brain Pathol [epub ahead of print], 2015

40. Phillips HS, Kharbanda S, Chen R, Forrest WF, Soriano RH, Wu TD, et al: Molecular subclasses of high-grade glioma predict prognosis, delineate a pattern of disease progression, and resemble stages in neurogenesis. Cancer Cell 9:157-173, 2006

41. See AP, Han JE, Phallen J, Binder Z, Gallia G, Pan F, et al: The role of STAT3 activation in modulating the immune microenvironment of GBM. J Neurooncol 110:359-368, 2012

42. Singh A, Settleman J: EMT, cancer stem cells and drug resistance: an emerging axis of evil in the war on cancer. Oncogene 29:4741-4751, 2010

43. Solecki DJ, Liu XL, Tomoda T, Fang Y, Hatten ME: Activated Notch2 signaling inhibits differentiation of cerebellar granule neuron precursors by maintaining proliferation. Neuron 31:557-568, 2001

44. Song Y, Li J, Zhu Y, Dai Y, Zeng T, Liu L, et al: MicroRNA-9 promotes tumor metastasis via repressing E-cadherin in esophageal squamous cell carcinoma. Oncotarget 5:11669-11680, 2014

45. Subramanian A, Tamayo P, Mootha VK, Mukherjee S, Eb- ert BL, Gillette MA, et al: Gene set enrichment analysis: a knowledge-based approach for interpreting genome-wide expression profiles. Proc Natl Acad Sci U S A 102:1554515550,2005

46. Sumazin P, Yang X, Chiu HS, Chung WJ, Iyer A, LlobetNavas D, et al: An extensive microRNA-mediated network of RNA-RNA interactions regulates established oncogenic pathways in glioblastoma. Cell 147:370-381, 2011

47. Tell RW, Horvath CM: Bioinformatic analysis reveals a pattern of STAT3-associated gene expression specific to basallike breast cancers in human tumors. Proc Natl Acad Sci U S A 111:12787-12792, 2014

48. Verhaak RG, Hoadley KA, Purdom E, Wang V, Qi Y, Wilkerson MD, et al: Integrated genomic analysis identifies clinically relevant subtypes of glioblastoma characterized by abnormalities in PDGFRA, IDH1, EGFR, and NF1. Cancer Cell 17:98-110, 2010

49. Vivanco I, Rohle D, Versele M, Iwanami A, Kuga D, Oldrini $\mathrm{B}$, et al: The phosphatase and tensin homolog regulates epidermal growth factor receptor (EGFR) inhibitor response by targeting EGFR for degradation. Proc Natl Acad Sci U S A 107:6459-6464, 2010

50. Wang Z, Li Y, Kong D, Banerjee S, Ahmad A, Azmi AS, et al: Acquisition of epithelial-mesenchymal transition phenotype of gemcitabine-resistant pancreatic cancer cells is linked with activation of the notch signaling pathway. Cancer Res 69:2400-2407, 2009

51. Wu H, Huang M, Cao P, Wang T, Shu Y, Liu P: MiR-135a targets JAK2 and inhibits gastric cancer cell proliferation. Cancer Biol Ther 13:281-288, 2012

52. Wu N, Liu J, Zhao X, Yan Z, Jiang B, Wang L, et al: Cardamonin induces apoptosis by suppressing STAT3 signaling pathway in glioblastoma stem cells. Tumour Biol 36:96679676, 2015

53. Yan S, Zhou C, Zhang W, Zhang G, Zhao X, Yang S, et al: beta-Catenin/TCF pathway upregulates STAT3 expression in human esophageal squamous cell carcinoma. Cancer Lett 271:85-97, 2008

54. Yan YR, Xie Q, Li F, Zhang Y, Ma JW, Xie SM, et al: Epithelial-to-mesenchymal transition is involved in BCNU resistance in human glioma cells. Neuropathology 34:128-134, 2014

55. Yang X, Liang L, Zhang XF, Jia HL, Qin Y, Zhu XC, et al: MicroRNA-26a suppresses tumor growth and metastasis of human hepatocellular carcinoma by targeting interleukin6-Stat3 pathway. Hepatology 58:158-170, 2013

56. Yokogami K, Wakisaka S, Avruch J, Reeves SA: Serine phosphorylation and maximal activation of STAT3 during CNTF signaling is mediated by the rapamycin target mTOR. Curr Biol 10:47-50, 2000

57. Yu H, Kortylewski M, Pardoll D: Crosstalk between cancer and immune cells: role of STAT3 in the tumour microenvironment. Nat Rev Immunol 7:41-51, 2007

58. Yue P, Turkson J: Targeting STAT3 in cancer: how successful are we? Expert Opin Investig Drugs 18:45-56, 2009

59. Zavadil J, Cermak L, Soto-Nieves N, Böttinger EP: Integration of TGF-beta/Smad and Jagged1/Notch signalling in epithelial-to-mesenchymal transition. EMBO J 23:1155-1165, 2004

60. Zhang YW, Wang LM, Jove R, Vande Woude GF: Requirement of Stat 3 signaling for HGF/SF-Met mediated tumorigenesis. Oncogene 21:217-226, 2002

\section{Disclosures}

The authors report no conflict of interest concerning the materials or methods used in this study or the findings specified in this paper. 


\section{Author Contributions}

Conception and design: Wu, Cheng, Zhang, Cai, M Li. Acquisition of data: Wu, Zhang, Ren, Jiang, Han, Cai. Analysis and interpretation of data: Cheng, Zhang, Yang Liu, Cai, M Li. Drafting the article: $\mathrm{Wu}$, Cheng, Zhang, Jiang. Critically revising the article: Wu, Zhang. Reviewed submitted version of manuscript: $\mathrm{Wu}$, Zhang, $\mathrm{Hu}, \mathrm{Bao}$. Approved the final version of the manuscript on behalf of all authors: Wu. Statistical analysis: Cheng, Zhang, Han, Yang Liu, Cai, M Li, Wang, Hu, Q Li. Administrative/technical/material support: Cheng, Zhang, Ren, Wang, Yanwei Liu, Hu, Q Li, Yang, Bao. Study supervision: Han, Yanwei Liu, Hu, Yang.

\section{Supplemental Information}

Online-Only Content

Supplemental material is available with the online version of the article.

Supplementary Data. http://thejns.org/doi/suppl/10.3171/ 2015.11.JNS15432.

\section{Correspondence}

Anhua Wu, Department of Neurosurgery, The First Hospital of China Medical University, Nanjing Street 155, Heping District, Shenyang 110001, China. email: cmuwuanhua@aliyun.com. 